EchoGéo

\title{
Hommage à Matthieu Giroud
}

\section{Matthieu Delage and Serge Weber}

\section{(2) OpenEdition}

Journals

Electronic version

URL: https://journals.openedition.org/echogeo/14640

DOI: 10.4000/echogeo.14640

ISSN: 1963-1197

\section{Publisher}

Pôle de recherche pour l'organisation et la diffusion de l'information géographique (CNRS UMR 8586)

\section{Electronic reference}

Matthieu Delage and Serge Weber, "Hommage à Matthieu Giroud", EchoGéo [Online], 36 | 2016, Online since 30 June 2016, connection on 11 August 2021. URL: http://journals.openedition.org/echogeo/ 14640 ; DOI: https://doi.org/10.4000/echogeo.14640

This text was automatically generated on 11 August 2021

EchoGéo est mis à disposition selon les termes de la licence Creative Commons Attribution - Pas d'Utilisation Commerciale - Pas de Modification 4.0 International (CC BY-NC-ND) 


\title{
Hommage à Matthieu Giroud
}

\author{
Matthieu Delage and Serge Weber
}

1 Echogéo a publié deux fois dans la rubrique Sur l'écrit, des contributions de Matthieu Giroud, l'une portant sur la place des commerçants dans les quartiers populaires affectés par un changement social et urbain (Berroir et al., 2015) et l'autre étant une interview retraçant les conditions de publication de la traduction qu'il a coordonnée d'un ouvrage incontournable de David Harvey, Paris, capitale de la modernité (Weber, 2013).

2 La revue Echogéo a souhaité lui rendre à son tour hommage, au lendemain de la journée organisée en sa mémoire à l'Université Paris-Est Marne-la-Vallée le 27 mai 2016, six mois après sa disparition, qui a rassemblé plus de deux cent personnes. Cette journée avait été préparée de manière collective, associant des personnes qui ont travaillé avec lui dans les différents laboratoires ou groupes de recherche, en particulier au laboratoire Migrinter et à l'université de Poitiers, au CERAMAC et à l'université de Clermont-Ferrand, au LAVUE, au laboratoire ACP et au sein du Labex Futurs urbains à l'université Paris-Est Marne-la-Vallée, sans oublier les collègues étrangers, que ce soit au Portugal, au Brésil ou en Grande-Bretagne, qui ont collaboré avec lui au sein de programmes de recherche variés, ni les auteurs, éditeurs et traducteurs avec qui il a fait aboutir plusieurs publications collectives de première importance. Autour de thèmes tels que "Villes et mobilités", "Changement social urbain et résistances habitantes» ou encore "Peut-on faire une géographie engagée", incluant des personnes avec qui Matthieu avait étudié ou qui avaient étudié avec lui, nombreux ont été les témoignages retraçant les étapes de son parcours, ses manières de faire de la recherche, d'enseigner et d'écrire, dans une perspective non seulement d'hommage scientifique, mais aussi d'ouverture vers les horizons de développement de ses apports à la discipline géographique et plus généralement aux études urbaines.

3 Cette journée a été l'occasion, pour ses proches, de mieux connaître le milieu professionnel et les préoccupations scientifiques de Matthieu et de constater à quel point son travail a influencé et inspiré de nombreux collègues, jeunes et moins jeunes, en géographie ou dans d'autres disciplines concernées par l'urbain. Nous avons choisi de reproduire ici la conclusion de cette journée d'hommages. 
4 Les écrits de Matthieu Giroud sont nombreux et diversifiés, comme en témoigne sa bibliographie, particulièrement étoffée ${ }^{1}$. Une partie importante, peut-être la plus monumentale, de sa pratique de l'écrit se place sous le signe de l'écriture collective beaucoup de ses publications les plus remarquables sont le fruit d'un travail d'équipe où il a joué un rôle essentiel en termes de coécriture, relectures croisées, animation d'équipes de coauteurs et de traducteurs. L'autre trait saillant qui caractérise son activité de publication est sans doute le soin qu'il a eu d'établir des éditions critiques minutieuses d'auteurs, émanant en particulier hors du champ francophone, ainsi que son activité de traducteur (Giroud, Hernandez, 2012) : cela confirme dans le champ bibliographique ce qu'il était aisé de constater dans sa pratique professionnelle de géographe, à savoir une mission de passage, de transmission et de partage, entre les milieux scientifiques et professionnels, entre les langues, les pays et les continents.

\section{Arpenter pour se souvenir : pas de théorie critique sans pratique}

5 Les lieux d'observation que Matthieu a arpentés sont particulièrement variés: ses terrains ont été multiples, de Grenoble à Sao Paulo et Bogotá, de Lisbonne à Leeds, en passant par Paris, et en particulier la ville de Pantin. Il aura tour à tour interrogé ces villes sous l'angle des transformations de quartiers populaires et cosmopolites, du point de vue des habitants et des «continuités populaires» (Giroud, 2007a; 2011) ou des pratiques et de l'expérience de mobilité et de la migration (Dubucs et al., 2011 ; Giroud, Mainet, Edouard, 2012 ; Imbert et al., 2014).

6 S'il ne fallait retenir qu'une entrée pour caractériser son objet de préoccupation, c'est le «changement urbain", et pas n'importe où: dans les quartiers anciens des métropoles, centraux ou péricentraux, en particulier ceux qui sont touchés par ce qu'on appelle la gentrification (Giroud, 2013 ; Le Blanc et al., 2014 ; Chabrol et al., 2016). Le changement urbain peut être opéré selon les cas par les aménagements liés aux grands événements culturels ou sportifs (Giroud, 2007b ; Giroud, Grésillon, 2011), par les politiques publiques de "régénération urbaine", ou par l'arrivée de nouveaux habitants qui contribuent par leur présence à modifier l'équilibre des rapports sociaux locaux.

7 Dans cette famille de plus en plus riche de recherches sur le changement urbain, Matthieu se distinguait par son attention aux implications pour les habitants « déjà là ", qui peuvent pâtir ou parfois indirectement bénéficier de ces mutations. Des espaces où « les anciens habitants peuvent vivre » - envers et contre tout (Asselin et al., 2005). Et pourtant, «face aux bulldozers, les habitants sont impuissants ». Tous les témoignages des collègues avec qui il a travaillé s'accordent à relever sa distance prudente, voire une forme de scepticisme, vis-à-vis des théories qui valideraient un " prêt-à-penser » en termes de modèles urbains. Théoriser, c'est entretenir une relation avec les faits, voilà une des postures de Matthieu qui a été rappelée lors de cette journée par de nombreux intervenants. Même s'il a été toujours très attentif aux théories urbaines qui se sont succédées, dont certaines se sont imposées plus ou moins durablement, notamment les conceptualisations de Neil Brenner, Andy Merrifield, John Allen, Saskia Sassen, Marcelo Lopez de Susa ou de Christian Schmidt, Matthieu n'a eu de cesse de rappeler que les théorisations, qu'elles soient critiques et/ou subversives, ne pouvaient se passer de l'aller-retour indispensable dans tout travail sérieux avec 
l'empirie, avec le terrain, avec le point de vue des habitants de celles et ceux qui faisaient l'expérience quotidienne, parfois à leur corps défendant, des mutations urbaines. C'est en particulier pour cette raison qu'il a porté son attention aux « résistances » des habitants, qu'elles soient organisées, publicisées et revendiquées ou au contraire discrètes, spontanées et individuelles (Giroud, 2005). Une approche critique des changements urbains et des modèles qui les façonnent passait pour lui nécessairement par une pratique critique, par une manière d'être impliqué sur et dans le terrain, aux côtés des habitants, des usagers, en étant attentif à leurs pratiques.

Matthieu a eu l'occasion de travailler dans de nombreux environnements scientifiques : à Grenoble, à Lyon, à Poitiers, à Clermont-Ferrand, à Paris ainsi que dans plusieurs réseaux internationaux de chercheurs. Il s'est frotté à de multiples manières de faire, de parler, de faire de la recherche, de penser, d'organiser le temps, d'écrire, de lire et d'être relu... et il a fait l'expérience quotidienne de différentes cultures scientifiques : en particulier celles qui appréhendent le temps long, l'histoire urbaine. La microstoria l'a par exemple passionné, et l'a inspiré pour saisir la mémoire des lieux et plus encore la mémoire des anonymes, pour explorer les formes de savoirs non savants d'anonymes qui ont leur interprétation des transformations de la ville. Dans cette perspective, il a utilisé avec une rigueur imperturbable une méthode cherchant à repérer comment les enquêté-es "se souviennent", s'appuyant sur la matrice biographique qui, d'une certaine manière, procède nécessairement $d u$ « je me souviens » (Dureau et al., 2014). Ceci révèle en creux une des préoccupations de Matthieu: l'organisation du temps, organisation minutieuse des activités, des lieux, des «tranches de vie " associées à des lieux, à des pratiques et à des conditions de vie dans les biographies.

9 Il a travaillé "dans les pas» de plusieurs personnes: il y a indéniablement des filiations, il a néanmoins tracé sa propre voie, que d'autres ont commencé et vont continuer à suivre.

10 Que ce soit sur le terrain ou dans ses cours, en bon géographe, il était attentif au visible, au paysage (Giroud, 2008). Comment regardait-il le «paysage»? ll y cherchait les traces des mémoires en voie de disparaitre, déjà éraflées, menacées de disparition qui le touchaient. Il était attentif à «l'envers du décor » (cette expression était centrale dans un de ses cours de Licence sur le paysage, qu'il avait construit à partir de l'exemple de Singapour). Et il avait une certaine jubilation à démasquer l'esthétisation de la face mise en scène du paysage urbain, de la face montrée, en tant qu'elle recèle tout un monde de rapports sociaux qu'il s'attachait à documenter.

11 Finalement, Matthieu a contribué à étayer ce que peut être une approche critique. Être critique, c'est documenter, de la manière la plus sérieuse possible, accumuler les preuves de ce qui ne convaincrait pas les incrédules, c'est dévoiler, démasquer. C'est informer, donner les clefs pour savoir, pour comprendre. Son travail acharné de traduction, d'édition critique, de publicisation (au sens de rendre public) confirme une chose : ce qui a fait bouger ses propres lignes, et qui d'une certaine manière l'a rendu meilleur, il a voulu le partager le plus largement possible.

\section{De l'engagement à la poétique du terrain}

12 Ce goût des villes, il l'a développé en tant que chercheur bien sûr, mais aussi comme voyageur et tout simplement comme habitant. Ce goût lui a donné un rapport fin au terrain, très longuement pratiqué, par l'observation, la marche, l'attente, les allers et 
retours, les discussions sans fin avec des patrons de café. Passer un tel temps, accepter une telle lenteur pour la recherche, tous les chercheurs ne le font pas... Cela révèle en creux un rapport au temps particulier. Une acceptation de ce que d'autres ressentiraient comme l'ennui, même s'il ne nous semble pas qu'il se soit jamais ennuyé sur le terrain. Une acceptation de la présence, de soi-même, des autres. Où qu'il soit, il habitait toujours le lieu où il se trouvait. Pour quelqu'un qui pratiquait la notion "d'habiter", on peut dire qu'il habitait ses terrains. Il se fondait dans la foule, se comportait comme un autre parmi les autres. Et cette manière de se «fondre dans la foule " plutôt que de se camoufler en embuscade, c'était certainement une manière d'accepter son altérité, sa position forcément décalée, ses pas de côté, c'était peut-être un moyen non seulement de s'ouvrir à l'altérité des autres, de s'approcher d'eux, de provoquer ce face-à-face décisif qu'est l'entretien, mais, précisément en faisant cela, en acceptant de se voir dans le regard des autres, d'accepter sa propre condition.

Observer, imaginer, se mettre à la place de l'autre, le suivre... il y a quelque chose, dans sa sensibilité au terrain, qui pourrait relever d'univers comme ceux de Tardi, du polar, de la bande dessinée, de la série, du cinéma bien sûr - en particulier des films de science-fiction comme Metropolis, Blade Runner, ou encore Le Cinquième élément ou Elysium, qu'il mobilisait dans ses dispositifs pédagogiques toujours millimétrés. Ce passage par la fiction lui permettait d'opérer une transposition du réel présent, en fonction de ce qu'on y décèle comme devenir probable, pour mieux l'identifier. Parce qu'il avait cette "reine des facultés ", l'imagination, de supposer ce que le devenir en cours pouvait donner de pire ou de meilleur, il avait à cœur de comprendre ce que les gens, aux prises avec les amorces d'un changement structurel, ressentaient. Et pour ce faire, il laissait une place à l'onirisme dans la recherche d'hypothèses et d'interprétations - comme en témoigne son attention aux univers féériques de Hayao Miyazaki (Giroud, Milhaud, 2005) ou encore des références récurrentes à des poètes comme Pablo Neruda ou Francis Ponge.

On serait donc tenté de se demander s'il n'y avait pas, dans sa pratique de recherche, un désir de scénariser quelque chose des vies autres, anonymes, s'il ne cherchait pas à les désanonymer, à les faire entendre. Ceci renvoie à ce qu'on pourrait appeler une poétique du terrain, une manière poétique d'être présent aux côtés des personnes qu'il regardait marcher dans la rue, s'accouder au comptoir, investir les espaces publics. Dans ses observations, il notait et caractérisait les ambiances sonores, mais il ne les a pas (à notre connaissance) portées pleinement au rang d'objet d'étude. Il est certain qu'il écoutait, comme d'autres géographes "la petite musique des territoires" (Canova, Bourdeau, Soubeyran, 2014). À suivre les trajectoires des uns et des autres, il notait le contrepoint des voix, la polyphonie, l'harmonie qui se dégage de présences et déplacements multiples qui s'offraient à son champ de vision. D'une certaine manière, son travail permet d'amplifier ces voix ténues. On aurait envie de comparer cette attitude à sa pratique, en tant que musicien, de la basse : un instrument qui ne se met pas au premier plan, soutient la mélodie des autres, assure la fondamentale, maintient la tenue de route dans le virage commun dans les modulations, et sur qui le groupe repose.

15 Amplifier ces voix, les rendre publiques, les élever au rang de savoir, orienter la recherche en ce sens, avec le plus grand sérieux possible, voilà un des sillages où il nous invite à le suivre. La journée du 27 mai nous l'a prouvé, nombreux sont les jalons qu'il a 
posés, ses façons de faire qui nous invitent à penser des alternatives. Et comme il ne travaillait pas seul, le passage de relais n'aura rien d'artificiel.

\section{BIBLIOGRAPHY}

Asselin O., Dureau F., Giroud M., Hamadi A., Marcadet Y., 2005. Access to urban resources as a spatial expression of social integration. In M. L. Fonseca, J. Malheiros, (dir.), Social integration and mobility: education, housing and health. IMISCOE Cluster B5 State of the art report, Lisbonne, Centro de Estudos Geográficos, Estudos para o Planeamento Regional e Urbano, nº 67, p. 49-65.

Berroir S., Clerval A., Delage M., Fleury A., Fol S., Giroud M., Raad L. Weber S., 2015. Commerce de détail et changement social urbain : immigration, gentrification, déclin. EchoGéo, n 33. http:// echogeo.revues.org/14353.

Canova N., Bourdeau P., Soubeyran O., 2014. La petite musique des territoires. Arts, espaces et sociétés. Paris, CNRS éditions, $242 \mathrm{p}$.

Chabrol M., Collet A., Giroud M., Launay L., Rousseau M., Ter Minassian H., 2016 (à paraître). Gentrifications. Paris, Les Prairies ordinaires.

Dubucs H., Dureau F., Giroud M., Imbert C., André-Poyaud I., Bahoken F., 2011. Les circulants entre métropoles européennes à l'épreuve de leurs mobilités. Une lecture temporelle, spatiale et sociale de la pénibilité. Articulo-Journal of Urban Research, $\mathrm{n}^{\circ} 7$.

Dureau F., Giroud M., Imbert C., 2014. La dynamique biographique des lieux de la circulation : une « affaire de famille»? In C. Imbert, H. Dubucs, F. Dureau, M. Giroud, D’une métropole à l'autre. Pratiques urbaines et circulations dans l'espace européen. Paris. Armand Colin, coll. Recherches, p. 305-362.

Gintrac C., Giroud M., (coord.), 2014. Villes contestées. Pour une géographie critique de l'urbain. Paris, Les prairies ordinaires, $416 \mathrm{p}$.

Giroud M., 2005. "Résister en habitant" : les luttes dans les quartiers populaires à l'épreuve du renouvellement urbain. Contretemps, $\mathrm{n}^{\circ} 13$, p. 49-58.

Giroud M., 2007a. Résister en habitant ? Renouvellement urbain et continuités populaires en centre ancien (Berriat Saint-Bruno à Grenoble et Alcântara à Lisbonne). Université de Poitiers, Thèse de doctorat en géographie sous la direction de F. Dureau, 518 p. http://tel.archives-ouvertes.fr/ tel-00200103/fr/

Giroud M., 2007b. Événement touristique et manières de vivre la ville. Le cas du championnat d'Europe des nations de football à Lisbonne. In P. Duhamel, R. Knafou (dir.), Les mondes urbains du tourisme. Paris, Belin, coll. Mappemonde, p. 88-103.

Giroud M., 2008. Illusion des façades et permanence ambiguë : la réhabilitation différentielle de la rue Coelho à Lisbonne. In C. Vallat, A. Delpirou, F. Maccaglia (dir.), Pérennité urbaine, ou la ville par delà des métamorphoses, Volume II : Turbulences. Paris, L'Harmattan, p. 165-181.

Giroud M., 2011. Usages des espaces rénovés et continuités populaires en centre ancien. Espaces et sociétés, $\mathrm{n}^{\circ}$ 144-145, p. 37-54. 
Giroud M., 2013. Habiter un quartier en voie de gentrification. Points de vue et pratiques d'habitants d'une opération de relogement à Lisbonne. In P. Harismendy, S. Gaudin (dir.), Vivre la rénovation urbaine. Les nouvelles pratiques d'habiter, Rencontres urbaines de Mazier. Saint-Brieuc, Corlet éditions, p. 187-205.

Giroud M., Grésillon B., 2011. Devenir capitale européenne de la culture : principes, enjeux et nouvelle donne concurrentielle. Cahiers de géographie du Québec, vol. 55, nº 155, p. 237-253.

Giroud M., Hernandez J. (dir.), 2012. Paris Capitale de la modernité, traduction de l'ouvrage de David Harvey. Paris, Les prairies ordinaires, $544 \mathrm{p}$.

Giroud M., Mainet H., Edouard J.-C. (dir.), 2012. Les mobilités spatiales dans les villes intermédiaires. Territoires, pratiques, régulations. Actes du colloque de Clermont-Ferrand, 25 et 26 novembre 2010, Clermont-Ferrand, Presses universitaires Blaise Pascal, 494 p.

Giroud M., Milhaud O., 2005. Sur le Château ambulant. Être ici et là-bas au même instant. EspacesTemps.net, http://espacestemps.net/document1384.html

Imbert C., Dubucs H., Dureau F., Giroud M., 2014. D'une métropole à l'autre. Pratiques urbaines et circulations dans l'espace européen. Paris, Armand Colin, coll. Recherches, 485 p.

Le Blanc A., Gervais-Lambony P., Giroud M., Pierdet C., Piermay J.-L., Rufat S. (dir.), 2014.

Métropoles en débat: (dé) constructions de la ville compétitive. Paris, Presses universitaires de Paris Ouest La Défense, $422 \mathrm{p}$.

Weber S., 2013. Traduire et lire Harvey : la pensée géographique par la brèche. Echogéo, $\mathrm{n}^{\circ} 22$. http://echogeo.revues.org/13284.

\section{NOTES}

1. On pourra se reporter à la liste complète de ses publications, consultable sur les sites des principaux laboratoires où il a travaillé, par exemple http://acp.u-pem.fr et http:// www.mshs.univ-poitiers.fr/migrinter/membre/Matthieu\%20Giroud.pdf. Nous remercions Françoise Dureau, Christophe Imbert et tous les autres collègues qui ont contribué à établir cette bibliographie.

\section{AUTHORS}

\section{MATTHIEU DELAGE}

Mattthieu Delage, matthieu.delage@u-pem.fr, est Maître de conférences à l'Université Paris-Est Marne-la-Vallée, Laboratoire ACP.

\section{SERGE WEBER}

Serge Weber, serge.weber@gmail.com, est Maître de conférences à l'Université Paris-Est Marnela-Vallée, Laboratoire ACP. 